\title{
Kv3 Potassium Conductance is Necessary and Kinetically Optimized for High-Frequency Action Potential Generation in Hippocampal Interneurons
}

\author{
Cheng-Chang Lien and Peter Jonas \\ Institute of Physiology, University of Freiburg, D-79104 Freiburg, Germany
}

Kv3 channels are thought to be essential for the fast-spiking (FS) phenotype in GABAergic interneurons, but how these channels confer the ability to generate action potentials (APs) at high frequency is unknown. To address this question, we developed a fast dynamic-clamp system $(\approx 50 \mathrm{kHz})$ that allowed us to add a Kv3 model conductance to CA1 oriens alveus (OA) interneurons in hippocampal slices. Selective pharmacological block of $\mathrm{Kv} 3$ channels by $0.3 \mathrm{~mm} 4$-aminopyridine or $1 \mathrm{~mm}$ tetraethylammonium ions led to a marked broadening of APs during trains of short stimuli and a reduction in AP frequency during 1 sec stimuli. The addition of artificial Kv3 conductance restored the original AP pattern. Subtraction of Kv3 conductance by dynamic clamp mimicked the effects of the blockers. Application of artificial Kv3 conductance also led to FS in OA interneurons after complete $\mathrm{K}^{+}$channel block and even induced FS in hippocampal pyramidal neurons in the absence of blockers. Adding artificial $\mathrm{Kv} 3$ conductance with altered deactivation kinetics revealed a nonmonotonic relationship between mean AP frequency and deactivation rate, with a maximum slightly above the original value. Insertion of artificial Kv3 conductance with either lowered activation threshold or inactivation also led to a reduction in the mean AP frequency. However, the mechanisms were distinct. Shifting the activation threshold induced adaptation, whereas adding inactivation caused frequency-dependent AP broadening. In conclusion, Kv3 channels are necessary for the FS phenotype of OA interneurons, and several of their gating properties appear to be optimized for high-frequency repetitive activity.

Key words: Kv3 channels; dynamic clamp; fast spiking; deactivation kinetics; $\mathrm{OA}$ interneurons; hippocampal slices; two electrode current clamp

\section{Introduction}

GABAergic interneurons in the mammalian cortex are able to generate action potentials (APs) at a very high frequency both in vitro (for review, see Connors and Gutnick, 1990) and in vivo (Ylinen et al., 1995; Jones et al., 2000). Two major lines of evidence suggest that sustained fast spiking (FS) is primarily conferred by delayed rectifier $\mathrm{K}^{+}$channels assembled from subunits of the Kv3 family (Rettig et al., 1992; Jan and Jan, 1997; Rudy and McBain, 2001). The first argument is the striking correlation between the expression of Kv3.1 and Kv3.2 subunits and the FS phenotype throughout the CNS (Du et al., 1996; Martina et al., 1998; Atzori et al., 2000; Lien et al., 2002; for review, see Rudy and McBain, 2001). The other piece of evidence is that the FS phenotype is severely impaired by the pharmacological block of Kv3 channels with either 4-AP or TEA (Martina et al., 1998; Erisir et al., 1999).

However, there are several difficulties with the hypothesis that $\mathrm{Kv} 3$ is the main determinant of the FS phenotype. First, the correlation between Kv3 expression and function is not absolutely strict, because some apparently non-FS cell types express Kv3

\footnotetext{
Received 0ct. 30, 2002; revised Dec. 27, 2002; accepted Dec. 30, 2002.

This work was supported by a scholarship from the Deutscher Akademischer Austauschdienst (C.-C.L.), Deutsche Forschungsgemeinschaft Grants J0-248/2-2 and SFB505/C5 (P.J.), and the Alexander-von-Humboldt Foundation. We thank Drs. B. Fakler, M. Heckmann, and G. Stuart for critically reading this manuscript; Drs. K. Haverkampf and I. Vida for advice; A. Blomenkamp and K. Winterhalter for technical assistance; and F. Heyde for secretarial help

Correspondence should be addressed to Dr. Peter Jonas, Institute of Physiology, University of Freiburg, HermannHerder Strasse 7, D-79104 Freiburg, Germany. E-mail: peter.jonas@physiologie.uni-freiburg.de.

Copyright $\odot 2003$ Society for Neuroscience $\quad 0270-6474 / 03 / 232058-11 \$ 15.00 / 0$
}

subunits (Chow et al., 1999; Betancourt and Colom, 2000). This could suggest that Kv3 is necessary but not sufficient for FS. Second, the genetic ablation of single Kv3 subunits, especially Kv3.1 and Kv3.3, leads to small changes in AP frequency and relatively mild behavioral phenotypes (Ho et al., 1997; Lau et al., 2000; Espinosa et al., 2001). This is surprising if Kv3 channels had a key role in FS, but may be explained by genetic redundancy (Martina et al., 1998; Espinosa et al., 2001; Porcello et al., 2002). Finally, the conclusion that a single type of conductance is of critical importance for the FS phenotype in interneurons is in apparent contrast to the current view on how characteristic AP patterns are generated in other types of neurons. In one model, the AP pattern is determined primarily by somatodendritic morphology (Mainen and Sejnowski, 1996). In another model, the AP pattern is generated by specific combinations of different conductances rather than a single conductance (Foster et al., 1993; Goldman et al., 2001). The relative importance of these different factors for FS in interneurons remains unclear.

To examine whether Kv3 channels are necessary and sufficient for FS and to identify the relevant gating properties, we sought to develop alternatives to the strategy of pharmacological or genetic elimination. We formulated a Hodgkin-Huxley (HH)-type model that accurately describes the gating of Kv3 channels in oriens alveus (OA) interneurons, a neuron type that generates APs at high frequency during depolarizing stimuli (Zhang and McBain, 1995a,b). We developed a fast dynamic-clamp system $(\approx 50 \mathrm{kHz})$ that allowed us to add this model conductance to real neurons (Sharp et al., 1993; Ma and Koester, 1996). Finally, we 
used this technique to determine quantitatively the functional impact of Kv3 channels in OA interneurons. The advantage of this approach is that it combines the realism of electrophysiological recordings with the flexibility and systematic nature of computational approaches.

\section{Materials and Methods}

Patch-clamp recording from $O A$ interneurons in hippocampal slices. Transverse hippocampal slices of $300 \mu \mathrm{m}$ thickness were cut from the brains of 17- to 22-d-old Wistar rats using a vibratome (Dosaka, Kyoto, Japan). Animals were killed by decapitation in agreement with national and institutional guidelines. Experiments were performed under visual control using infrared differential interference contrast videomicroscopy (Stuart et al., 1993; Lien et al., 2002). Interneurons with horizontal dendrites in stratum oriens alveus in the CA1 subfield were identified using the criteria reported previously (Martina et al., 2000; Lien et al., 2002). Only neurons with initial resting potentials more negative than $-55 \mathrm{mV}$ were accepted. The recording temperature was $21-24^{\circ} \mathrm{C}$.

Recording from nucleated patches. Nucleated patch recordings were made as described previously (Martina and Jonas, 1997; Martina et al., 1998; Lien et al., 2002), using an Axopatch 200A amplifier or a Multiclamp 700A amplifier (Axon Instruments, Foster City, CA). Signals were low-pass filtered at 5 or $6 \mathrm{kHz}$ (four-pole Bessel), and sampled at 10 or 20 kHz. A 1401plus interface-personal computer (PC) system (Cambridge Electronics Design, Cambridge, UK) was used for stimulus generation and data acquisition. Nucleated patches were held at $-90 \mathrm{mV}$. Pulse sequences were generated by homemade programs. Leakage and capacitive currents were subtracted on-line using a " $P /-4$ " procedure (Martina and Jonas, 1997). Pulse sequences were applied every 4-10 sec. Kv3 current components were isolated pharmacologically by subtracting traces in the presence of either $0.3 \mathrm{~mm} 4$-AP or $0.5-1 \mathrm{~mm}$ TEA from traces in the absence of blockers.

Analysis of nucleated patch data. To take the effects of low-pass filtering into account, current traces were shifted by $-100 \mu \mathrm{sec}(\approx 340 \mu \mathrm{sec}$ divided by corner frequency) against the pulse sequence. To determine the activation time constant, $\mathrm{K}^{+}$currents during test pulses were fitted with an exponential function $A\left[1-\exp \left(-[t-\delta] / \tau_{\text {act }}\right)\right]$ for $t \geq \delta$, where $A$ is the current amplitude, $\tau_{\text {act }}$ is the activation time constant, and $\delta$ is a delay. Exponential functions without a delay and high-power exponentials $A\left[1-\exp \left(-t / \tau_{\mathrm{act}}\right)\right]^{a}(a=2,3$, or 4$)$ were also tested but in most cases gave worse fits to the data. To determine the deactivation time constant, tail currents after test pulses were fitted with an exponential function $A \exp \left(-t / \tau_{\text {deact }}\right)+B$, in which $\tau_{\text {deact }}$ is the deactivation time constant and $B$ represents an offset. Data points $\leq 200 \mu \mathrm{sec}$ after the voltage step were excluded from the fit. Fitting was made using homemade programs or Mathematica 4.1 (Wolfram Research, Champaign, IL), using nonlinear least-squares algorithms.

Activation curves were determined by two alternative methods. First, chord conductance was calculated from the outward current in $2.5 \mathrm{~mm}$ external $\left[\mathrm{K}^{+}\right]$, assuming ohmic behavior and a reversal potential of -95 $\mathrm{mV}$ (Martina et al., 1998). Second, activation curves were obtained directly by plotting the amplitude of the inward tail current in $25 \mathrm{~mm}$ external $\left[\mathrm{K}^{+}\right]$(amplitude of the fitted exponential $300 \mu$ sec after the voltage step) against the voltage of the conditioning pulse. In both cases, data were normalized to the respective mean at 50-70 $\mathrm{mV}$.

Description of Kv3 channel gating with an HH-type model. When modeling the gating of $\mathrm{Kv} 3$ in $\mathrm{OA}$ interneurons, two observations had to be accounted for. First, activation and deactivation time constants were very similar when measured at the same potential ( -10 to $10 \mathrm{mV})$. Second, activation occurred with a mean delay of $\approx 0.3 \mathrm{msec}$, which was relatively independent of voltage. We therefore used a modified HH-type model with a single "gating particle" and a delay in activation that accounts for transitions between closed states. This model described the data adequately (see Fig. $1 E, F$ ). In contrast, the original $\mathrm{HH}$ model with four gating particles implies that the deactivation $\tau$ is up to four times faster than the activation $\tau$ at the same potential (Hille, 2001).

The activation rate $\alpha$ was represented by the following equation:

$$
\alpha=a \times[-(V+b)] /(\exp [-(V+b) / c]-1) .
$$

The deactivation rate $\beta$ was described by the following equation:

$$
\beta=d \times \exp (-V / e),
$$

where $a-e$ describe the values of the rates and their voltage dependence, respectively (Hodgkin and Huxley, 1952). The steady-state activation parameter was obtained as $n_{\infty}=\alpha /(\alpha+\beta)$, and the activation time constant as $\tau=(\alpha+\beta)^{-1}$. The parameters $a-e$ were then varied to minimize the total sum of squares of differences between the model and all experimental data using the FindMinimum procedure of Mathematica. Weight factors were set arbitrarily according to the expected precision in the experimental estimates. The best fit of the experimental data was obtained with $a=0.0189324 \mathrm{msec}^{-1}, b=-4.18371 \mathrm{mV}, c=$ $6.42606 \mathrm{mV}, d=0.015857 \mathrm{msec}^{-1}$, and $e=25.4834 \mathrm{mV}$.

Fast dynamic-clamp system. To add artificial $\mathrm{K}^{+}$conductance to real neurons, a fast dynamic-clamp system was developed (Sharp et al., 1993; Ma and Koester, 1996) (see Fig. 2B). The system consisted of a digital signal processor (DSP) board (60 MHz, Texas Instruments TMS320C32; Innovative Integration, Westlake Village, CA). Both the analog-digital converter (ADC, 16 bit; conversion time, $<10 \mu \mathrm{sec}$; anti-aliasing filter set to corner frequencies, $>100 \mathrm{kHz}$ ) and the digital-analog converter (DAC, 16 bit; output bandwidth, $200 \mathrm{kHz}$ ) were optimized for speed. The DSP board was inserted into a PC and driven by a homemade program written in $\mathrm{C}$ and compiled using a Texas Instruments $\mathrm{C}$ compiler for the TMS320C3x processor family.

During initialization of the DSP board, tables of $\alpha(V)$ and $\beta(V)$ were generated for the channel model (at $0.1 \mathrm{mV}$ resolution) and stored in the static random-access memory of the board. In the dynamic-clamp mode, the voltage of the cell $(V)$ was read from the ADC, and the corresponding values of $\alpha(V)$ and $\beta(V)$ were obtained by linear interpolation from the tables. $n_{\infty}$ was calculated as $\alpha /(\alpha+\beta)$, and $\tau$ was calculated as $(\alpha+\beta)^{-1}$. To implement the delay of activation $\delta, \alpha$ was taken from a history table at the time point $t-\delta$; $\delta$ was assumed to be $0.3 \mathrm{msec}$ throughout. For each time step $\Delta t$, the activation parameter $(n)$ was updated as follows:

$$
n(t+\Delta t)=n(t)+\left[n_{\infty}-n(t)\right] / \tau \times \Delta t .
$$

Finally, the $\mathrm{K}^{+}$current was calculated as follows:

$$
I(t+\Delta t)=G_{\max } \times n(t+\Delta t) \times\left(V-V_{\mathrm{K}}\right),
$$

where $G_{\max }$ is the maximal $\mathrm{K}^{+}$conductance and $V_{\mathrm{K}}$ is the assumed reversal potential ( $-95 \mathrm{mV}$ throughout). The calculation was performed in a timer- and interrupt-driven procedure, using the commands enableclock, installintvector, and enableinterrupt of the Innovative Integration PCI32C language supplement library. The dynamic-clamp system was run at a frequency of $\approx 50 \mathrm{kHz}$ (i.e., $\Delta t \approx 20 \mu \mathrm{sec}$ ).

In some dynamic-clamp experiments, gating properties were altered compared with the original Kv3 channels. First, the deactivation time course was changed by multiplying $\tau^{-1}(V)$ by a constant factor $f_{\beta}(0.1-$ 10) for $n_{\infty}-n(t)<0$. This strategy leads to a selective change in deactivation kinetics without any influence on activation kinetics. Second, the voltage dependence of both $n_{\infty}(V)$ and $\tau(V)$ was shifted by a constant offset $(-10$ to $-30 \mathrm{mV})$. Third, an inactivation process was implemented by multiplying the right side of Equation 4 by the inactivation variable $h$. The time constant of inactivation onset was $30 \mathrm{msec}$, the time constant of recovery was $1 \mathrm{sec}$ (Geiger and Jonas, 2000), and the steadystate inactivation parameter $\left(h_{\infty}\right)$ was represented by a Boltzmann function with a midpoint potential $\left(V_{1 / 2}\right)$ of $-78.5 \mathrm{mV}$ and a steepness factor of $6 \mathrm{mV}$ (Lien et al., 2002). $h_{\infty}$ was normalized to the value of $h$ at $-70 \mathrm{mV}$ to keep constant the number of available $\mathrm{K}^{+}$channels at rest. The accuracy of the dynamic-clamp system was verified by applying voltageclamp pulse protocols to the system (see Fig. 2C).

In a separate set of dynamic-clamp experiments, we varied the value of $\delta$ between 0 and $1 \mathrm{msec}$. This change led to a slight alteration of the shape of the AP but gave very similar results for AP frequency. In another set of measurements, we implemented a serial multistate model with three closed states and one open state, linked by exponentially voltagedependent rates. This model gave results very similar to those of the HH-type model, but it was more difficult to alter individual gating properties selectively (Lien and Jonas, 2002). 
Addition and subtraction of Kv3 conductance. For dynamic-clamp experiments, a Multiclamp 700A amplifier (Axon Instruments) was used based on the voltage-follower principle (specified rise time, $<10 \mu \mathrm{sec}$ ) (Magistretti et al., 1998). To avoid series resistance $\left(R_{\mathrm{S}}\right)$ artifacts, dual current-clamp whole-cell recordings were made, allowing us to separate the current-feeding from the voltage-recording electrode (Roth and Häusser, 2001). Patch pipettes were pulled from borosilicate glass tubing ( $2.0 \mathrm{~mm}$ outer diameter; $0.5 \mathrm{~mm}$ wall thickness) and heat-polished before use. The pipette resistances ranged from 2 to $4 \mathrm{M} \Omega$. Both pipettes were positioned at the soma (distance between tips, $\approx 20 \mu \mathrm{m}$ ). Cells were held at $-70 \mathrm{mV}$ during the experiment; in some recordings (especially in the presence of blockers) a hyperpolarizing current was injected (10-50 $\mathrm{pA}$ ). Pipette capacitance was compensated for both pipettes, using values slightly lower than those determined previously in the cell-attached configuration $(5.5-7.5 \mathrm{pF}) . R_{\mathrm{S}}$ was compensated every 5-10 min using the automatic bridge balance (readouts after compensation were 7-10 $\mathrm{M} \Omega$ for the current-feeding electrode and 7-15 $\mathrm{M} \Omega$ for the voltage-recording electrode). The output signal of the amplifier was filtered by the internal four-pole Bessel filter at $24 \mathrm{kHz}$ and fed directly into the ADC of the DSP card. Additionally, the signal was filtered by an external eight-pole lowpass Bessel filter at $10 \mathrm{kHz}$ (Frequency Devices, Haverhill, MA) and recorded by the 1401 plus interface-PC system at a sampling frequency of $20 \mathrm{kHz}$.

For the addition of conductance after pharmacological block (rescue experiment; see Fig. 3), $G_{\max }$ was increased from 10 to 200 in $10 \mathrm{nS}$ steps, and the smallest $G_{\max }$ that gave $>95 \%$ restoration of the half-duration of the 10th $\mathrm{AP}$ in a $50 \mathrm{~Hz}$ train was chosen for subsequent recordings, unless specified differently. For the subtraction of conductance (see Fig. 4), $G_{\max }$ was changed from -10 to $-110 \mathrm{in}-10 \mathrm{nS}$ steps. Care was taken to avoid oversubtraction, which tends to destroy the recording by positive feedback loops (Sharp et al., 1993; Ma and Koester, 1996). After the subtraction experiment, 4-AP or TEA was applied, and the $G_{\max }$ value that gave the closest mimicry of the blocker effects on the 10th AP was chosen for subsequent analysis.

Analysis of dynamic-clamp data. The maximal rate of rise $d V / d t_{\max }$ was determined from the largest voltage difference between adjacent sample points in the AP rising phase. The AP amplitude was measured as the difference between the resting potential before stimulation and the peak. The AP half-duration was quantified as the time difference between the points at which the amplitude of the AP was half-maximal. The mean AP frequency was determined as the total number of APs divided by the duration $(1 \mathrm{sec})$ of the current pulse. The interspike intervals (ISIs) were determined from the time differences between the peaks of two consecutive APs. The instantaneous AP frequency was calculated as the inverse of a given ISI. Operationally, we define FS as the ability of a cell to generate trains of APs with a mean frequency of $\sim 30 \mathrm{~Hz}$ and little accommodation during $1 \mathrm{sec}$ current pulses (McBain and Fisahn, 2001; Lien et al., 2002). The coefficient of variation (CV) of ISIs was calculated as SD divided by mean. AP patterns were analyzed using homemade programs.

Solutions and chemicals. Slices were superfused with a physiological extracellular solution containing (in mM): $125 \mathrm{NaCl}, 25 \mathrm{NaHCO}_{3}, 2.5$ $\mathrm{KCl}, 1.25 \mathrm{NaH}_{2} \mathrm{PO}_{4}, 2 \mathrm{CaCl}_{2}, 1 \mathrm{MgCl}_{2}$, and 25 glucose. The $25 \mathrm{~mm}\left[\mathrm{~K}^{+}\right]$ solution contained (in $\mathrm{mM}$ ): $102.5 \mathrm{NaCl}, 25 \mathrm{NaHCO}_{3}, 25 \mathrm{KCl}, 1.25$ $\mathrm{NaH}_{2} \mathrm{PO}_{4}, 2 \mathrm{CaCl}_{2}, 1 \mathrm{MgCl}_{2}$, and 25 glucose. Both solutions were equilibrated with $95 \% \mathrm{O}_{2}$ and $5 \% \mathrm{CO}_{2}$. The $\mathrm{K}^{+}$channel blockers 4-AP (Sigma, St. Louis, MO) and TEA (Merck, Darmstadt, Germany) were applied externally via bath superfusion. Recording pipettes were filled with an internal solution containing (in mM): $120 \mathrm{~K}$-gluconate and $20 \mathrm{KCl}$ (in the majority of whole-cell recordings) or $140 \mathrm{KCl}$ (in all nucleated patch experiments and some whole-cell recordings), 10 EGTA, $2 \mathrm{MgCl}_{2}, 2$ $\mathrm{Na}_{2} \mathrm{ATP}$, and 10 HEPES, pH adjusted to 7.3 with $\mathrm{KOH}$. In the majority of experiments, $0.3-0.5 \%$ biocytin was added to visualize cell morphology (Lien et al., 2002). In 7 of 18 cells filled, the axon could be traced to the stratum lacunosum-moleculare. In 11 of 18 cells, the staining ended in the stratum radiatum, pyramidale, or oriens, presumably caused by the cutting of the axon or insufficient filling. Chemicals were obtained from Merck, Sigma, Riedel-de-Haën (Seelze, Germany), Gerbu (Gaiberg, Germany), or Molecular Probes (Eugene, OR).
Conventions. Data are reported as mean \pm SEM; error bars in the figures also represent SEM (they are shown only when they exceed the size of the symbol). In panels showing superimposed APs, the black traces were obtained under control conditions and in the presence of blockers, and the gray traces were recorded after the addition or subtraction of Kv3 by dynamic clamp. The dynamic-clamp current is depicted below the AP traces throughout. Statistical significance was assessed using a Student's $t$ test for paired or unpaired samples at a given significance level $(p)$. The data included in this study were obtained from 32 nucleated patch recordings, partly taken from Lien et al. (2002), and 37 two electrode whole-cell recordings ( 29 from OA interneurons and eight from pyramidal neurons).

\section{Results \\ Gating model of Kv3 channels and its implementation in a dynamic-clamp system}

To examine the importance of Kv3 channels for the FS phenotype in OA interneurons, we characterized the gating of these channels quantitatively. Activation and deactivation kinetics were measured under ideal voltage-clamp conditions in nucleated patches (Fig. 1). The Kv3 component was isolated pharmacologically by subtracting currents in the presence of either $0.3 \mathrm{~mm} 4$-AP or 0.5 or $1 \mathrm{~mm}$ TEA from currents under control conditions (Martina et al., 1998; Lien et al., 2002). Recordings were made in both a normal $(2.5 \mathrm{~mm})$ and elevated $(25 \mathrm{~mm})$ external $\mathrm{K}^{+}$concentration, to increase the precision in the measurement of deactivation kinetics. Based on these experiments, activation curves and plots of activation and deactivation time constants as a function of voltage were constructed (Fig. 1E,F). In agreement with the known properties of Kv3 channels (Rudy and McBain, 2001), the midpoint potential of the activation curve was $-12.4 \mathrm{mV}$, and the relationship between time constants and voltage was bellshaped, reaching submillisecond values at negative voltages (Fig. $1 E, F)$.

We next attempted to describe activation curves and the voltage dependence of time constants with an HH-type model in which a delay was incorporated (Fig. 2A). The final model, which was generated by a variation of the parameters until the best fit was obtained, described the experimental data adequately (Fig. $1 E, F$, continuous curves). The model was then implemented into a dynamic-clamp system running at a frequency of $\approx 50 \mathrm{kHz}$ (Fig. $2 B$; see Materials and Methods). The time courses of the currents generated by the system in response to standard activation and deactivation pulse protocols were very similar to those measured in nucleated patches (compare Figs. $2 C$ and $1 A, C$ ), validating the implementation of our Kv3 channel model.

\section{Addition of $\mathrm{Kv} 3$ conductance by dynamic clamp after pharmacological block rescues the FS phenotype}

To examine whether Kv3 channels were necessary for FS in OA interneurons, we sought to develop approaches complementary to the pharmacological elimination (Martina et al., 1998; Erisir et al., 1999). Thus, we performed a "gain of function" experiment in which an artificial Kv3 conductance was added by dynamic clamp after pharmacological block (Fig. 3). We first examined the effects on the shape of single spikes evoked by brief current pulses. The block of Kv3 channels by either $0.3 \mathrm{~mm} 4$-AP or $1 \mathrm{~mm}$ TEA led to a substantial broadening of the first and the 10th AP evoked by a $50 \mathrm{~Hz}$ train in OA interneurons. The subsequent addition of artificial Kv3 conductance by the dynamic-clamp system resulted in a rescue of AP duration (Fig. 3A). To determine the amount of perisomatic Kv3 conductance necessary to restore the AP duration in a given $\mathrm{OA}$ interneuron, we varied the maximal conductance $\left(G_{\max }\right)$ and plotted the half-duration of the 10th AP against 


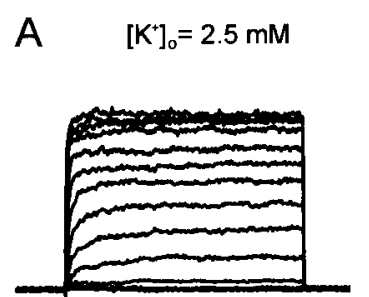

$$
\text { B } \quad[K]_{0}=25 \mathrm{mM}
$$

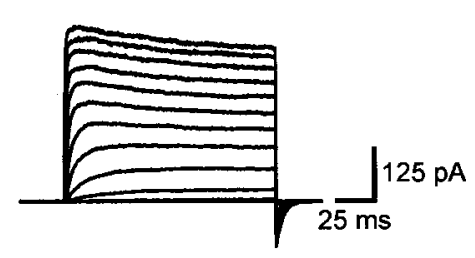

C $\quad\left[K^{+}\right]_{0}=2.5 \mathrm{mM}$

D $\left[\mathrm{K}^{+}\right]_{0}=25 \mathrm{mM}$
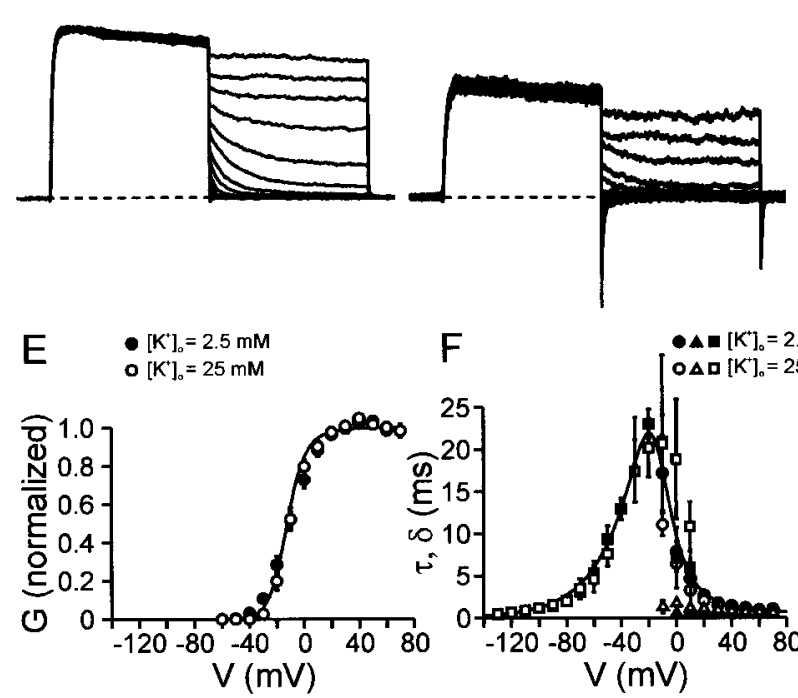

F $\quad \Delta \Delta\left[K \mathrm{~K}_{0}=2.5 \mathrm{mM}\right.$

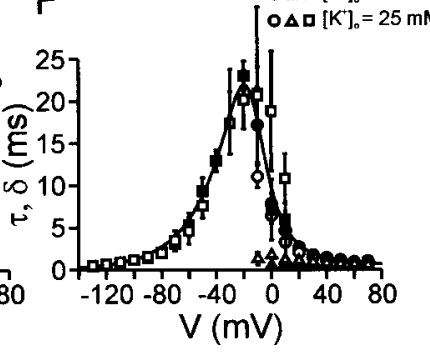

Figure 1. Gating of Kv3 channels in nucleated patches isolated from $0 A$ interneurons. $A-D$, Kv3 channel-mediated current in nucleated patches isolated by digital subtraction of traces in the presence of $1 \mathrm{~mm}$ TEA from traces under control conditions. A, B, Activation protocol $(-80$ to $70 \mathrm{mV}$ in $10 \mathrm{mV}$ increments). C, D, Deactivation protocol (conditioning pulse to $20 \mathrm{mV}$, steps to -130 to $10 \mathrm{mV}$ in $10 \mathrm{mV}$ increments). Dashed lines indicate baselines. $A, C$, Recordings in 2.5 mм external $\left[\mathrm{K}^{+}\right] . B, D$, Recordings in $25 \mathrm{~mm}$ external $\left[\mathrm{K}^{+}\right]$in a different patch. Traces, Single sweeps or averages of up to five sweeps. E, Kv3 channel activation curve. Filled circles indicate $2.5 \mathrm{~mm}$ external $\left[\mathrm{K}^{+}\right](n=10)$. Conductance $(G)$ was calculated from outward currents by ohmic correction. Open circles indicate $25 \mathrm{~mm}$ external $\left[\mathrm{K}^{+}\right](n=6)$. $G$ was obtained from the amplitudes of inward tail currents. F, Activation and deactivation kinetics. Activation $\tau$ (circles, $n=6-10)$, delay $\delta$ (triangles), and deactivation $\tau($ squares, $n=4-10$ ) are plotted against voltage. Filled symbols indicate $2.5 \mathrm{~mm}$ external $\left[\mathrm{K}^{+}\right]$. Open symbols indicate $25 \mathrm{~mm}$ external $\left[\mathrm{K}^{+}\right]$. Data in $2.5 \mathrm{~mm}$ external $\left[\mathrm{K}^{+}\right]$were partly taken from Lien et al. (2002). E, F, Continuous curves are predictions of the fitted HH-type model (Fig. 2A).

$G_{\max }$ (Fig. 3B). On average, the $G_{\max }$ required for complete rescue, corresponding to the crossing of the fitted curve with a line representing the control half-duration, was $122 \pm 13 \mathrm{nS}(n=17)$ (Fig. $3 B$ ). Around the crossing point, the half-duration was relatively insensitive to the value of $G_{\max }$.

We subsequently examined whether the addition of artificial Kv3 conductance after pharmacological block was able to rescue the FS pattern elicited by long current pulses (Fig. 3C,D), using a conductance $G_{\max }$ that restored (by $>95 \%$ ) the half-duration of the 10th AP evoked by a brief pulse train in the same cells (Fig. $3 A, B)$. Adding $0.3 \mathrm{~mm} 4$-AP or $1 \mathrm{~mm}$ TEA reduced the mean AP frequency from $41.1 \pm 1.5$ to $26.0 \pm 1.2 \mathrm{~Hz}$ ( $1 \mathrm{sec}, 0.5 \mathrm{nA}$ current pulse; $n=16$ ). A subsequent addition of artificial Kv3 conductance restored the FS phenotype (Fig. 3D). Under these conditions, the mean frequency was $43.0 \pm 1.4 \mathrm{~Hz}$, substantially higher than in the presence of blockers alone $(p<0.005)$ but not different from that under control conditions in the same cells $(p>$ $0.5)$. In conclusion, these results indicate that both the shape of a

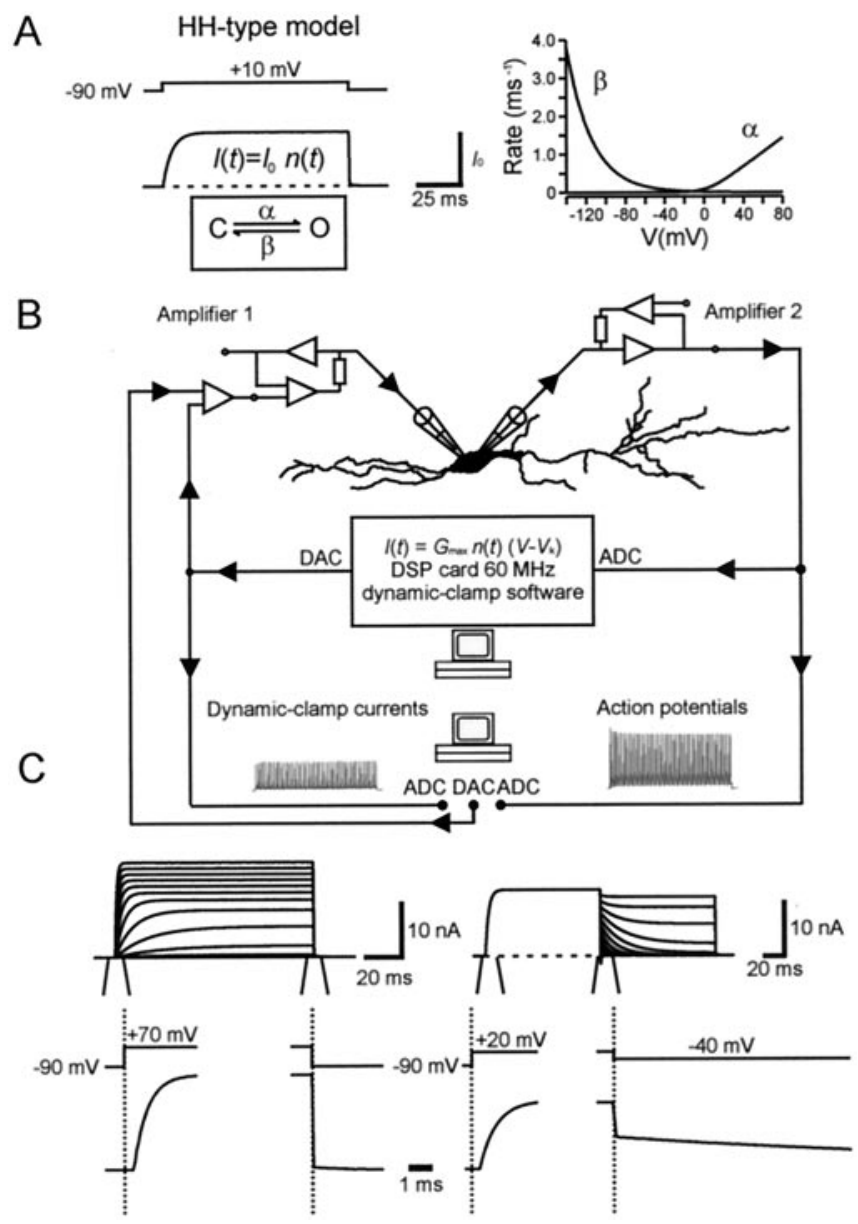

Figure 2. Design and performance of the dynamic-clamp system. A, HH-type model of Kv3 channels in $0 A$ interneurons. A simulated trace for a test pulse from -90 to $10 \mathrm{mV}$ (left) and rates $\alpha$ and $\beta$ as a function of potential (right) are shown. B, Schematic illustration of the dynamic-clamp system. Note that two pipettes were used, with both amplifiers in the currentclamp mode. Two electrode recording allowed us to record voltage signals without any $R_{S}$ artifacts. The membrane potential is recorded by amplifier 2 and digitized by the ADC of the DSP card. For every time step, the current is calculated from Equation 4 and converted into an analog-voltage signal by the DAC of the DSP card. Arrowheads indicate the direction of signal flow. For details, see Materials and Methods. C, Dynamic-clamp currents obtained in the openloop configuration with the HH-type model (delay, $0.3 \mathrm{msec} ; G_{\max }=100 \mathrm{nS}$ ). Left traces, Currents evoked by the activation protocol (test pulse, -80 to $70 \mathrm{mV}$ in $10 \mathrm{mV}$ increments) are shown. Right traces, Currents evoked by the deactivation protocol (conditioning pulse to $20 \mathrm{mV}$, steps to -130 to $10 \mathrm{mV}$ in $10 \mathrm{mV}$ increments) are shown. Dashed lines indicate baselines. Expanded activation and deactivation phases are also shown (bottom).

single spike and the FS phenotype during a train of spikes are rescued by the artificial Kv3 conductance.

\section{Subtraction of $\mathrm{Kv} 3$ conductance mimics the effects} of blockers

To obtain an alternative to the elimination of Kv3 by pharmacological methods (Martina et al., 1998; Erisir et al., 1999), we used the dynamic-clamp technique to subtract the Kv3 conductance electrically (Fig. 4) (Sharp et al., 1993). Subtraction of endogenous $\mathrm{Kv} 3$ conductance in OA interneurons led to a broadening of APs evoked by brief current pulses (Fig. 4A). As the amount of the subtracted conductance was increased, the half-duration of the single AP approached that observed in the presence of pharmacological blockers (Fig. $4 B$ ). On average, the value of $G_{\max }$ necessary to mimic the effects of pharmacological block, corresponding to the crossing of the fitted curve with a line represent- 
ing the half-duration in the presence of blockers, was $-141 \pm 22 \mathrm{nS}(n=10)$, similar to that required for the rescue of AP duration after block (122 $\pm 13 \mathrm{nS}$; see above). Both values were in approximate agreement with the mean perisomatic Kv3 conductance in OA interneurons (83.1 $\mathrm{nS})$. This value was estimated from the mean Kv3 conductance density in nucleated patches $\left(27.7 \pm 3.0 \mathrm{pS} \mu \mathrm{m}^{-2} ; n=32\right.$; test pulse, $70 \mathrm{mV} ; V_{\mathrm{K}}=-95 \mathrm{mV}$ ), and the area of the perisomatic compartment was determined from the whole-cell capacitance $\left(2956 \pm 730 \mu \mathrm{m}^{2}\right.$ with a specific membrane capacitance of $0.8 \mu \mathrm{F} \mathrm{cm}^{-2}$ ).

We also examined whether subtraction of endogenous Kv3 conductance altered the frequency of APs evoked by long current pulses (Fig. 4C,D), again using a conductance $G_{\max }$ that mimicked the effects of blockers on the half-duration of the 10th AP in the same cells. Subtraction of the Kv3 conductance led to a reduction in mean AP frequency from $43.4 \pm 2.2$ to $28.0 \pm 1.5 \mathrm{~Hz}$ ( $1 \mathrm{sec}, 0.5 \mathrm{nA}$ current pulse; $n=5 ; p<0.05)$, comparable with the effects of pharmacological block (21.2 \pm $4.4 \mathrm{~Hz} ; n=5$; $p>0.1$ ) (Fig. $4 C, D$ ). In conclusion, subtraction of Kv3 conductance by dynamic clamp to a large extent mimics the effects of pharmacological block by 4 -AP or TEA.

\section{$\mathrm{Kv} 3$ is sufficient for FS in two different types of cells}

The present results, in conjunction with previous data, show unequivocally that Kv3 channels are necessary for FS in interneurons. We wanted to go one step further and test whether Kv3 channels are sufficient for the generation of the FS phenotype. To examine this idea, we performed the rescue experiment in $\mathrm{OA}$ interneurons in the presence of saturating concentrations of $\mathrm{K}^{+}$channel blockers (Fig. $5 A-C$ ). In $5 \mathrm{~mm} 4$-AP plus $20 \mathrm{mM}$ TEA, which block $>95 \%$ of voltage-gated $\mathrm{K}^{+}$channels in OA interneurons (Lien et al., 2002), the AP duration was increased substantially (Fig. $5 A$ ), and the repetitive generation of APs during long current pulses was abolished completely (Fig. $5 B)$. Although this combination of blockers profoundly altered the AP pattern, the subsequent addition of the artificial Kv3 conductance, using a $G_{\max }$ that restored the half-duration of the 10th AP in the same cells, rescued the FS phenotype (Fig. $5 B, C$ ). Thus, after complete elimination of $\mathrm{K}^{+}$channels, Kv3 appears to be sufficient for the generation of the FS phenotype in OA interneurons. Surprisingly, the mean AP frequency was even higher after the addition of artificial Kv3 conductance than under control conditions in four of five cells (mean frequency, $38.6 \pm 2.9$ vs $45 \pm 4.2 \mathrm{~Hz}$; $1 \mathrm{sec}, 0.5 \mathrm{nA}$ current pulse; $n=5$ ).

Previous studies emphasized the importance of somatodendritic morphology in the generation of characteristic AP phenotypes (Mainen and Sejnowski, 1996). Therefore a stringent test of the Kv3 hypothesis of FS is whether the insertion of artificial Kv3 conductance is sufficient to generate FS in neurons with non-FS patterns (e.g., in CA1 pyramidal neurons) (Fig. 5D-F). Addition of artificial Kv3 conductance in CA1 pyramidal neurons ( $100 \mathrm{nS}$ ) led to a slight shortening of the first AP and a pronounced shortening of the 10th AP in a $50 \mathrm{~Hz}$ train of spikes (Fig. 5D). Similarly, the addition of artificial Kv3 converted the regular spiking of pyramidal neurons during long current stimuli into an FS phenotype (Fig. $5 E, F$ ). In CA1 pyramidal neurons, the mean AP frequency was $32.3 \pm 2.9 \mathrm{~Hz}$ after the addition of artificial Kv3 conductance, almost two times larger than that under control conditions in the same cells $(19.3 \pm 2.1 \mathrm{~Hz} ; n=8 ; p<0.005)$. Accordingly, Kv3 channels appear to be sufficient for the FS phenotype in both OA interneurons and CA1 pyramidal cells, despite the very different morphological properties of these cells.

\section{An intermediate deactivation rate is optimal for the FS phenotype}

Among all voltage-gated $\mathrm{K}^{+}$channels, $\mathrm{Kv} 3$ shows the fastest deactivation rate (Rudy and McBain, 2001). To examine quantitatively the impact of deactivation kinetics on the AP phenotype, we added artificial $\mathrm{Kv} 3$ channels with an altered deactivation rate (Fig. 6). Unlike the original Kv3, which rescued the sustained AP pattern (Figs. 3C, 6B1), altered Kv3 with either slower or faster 
A

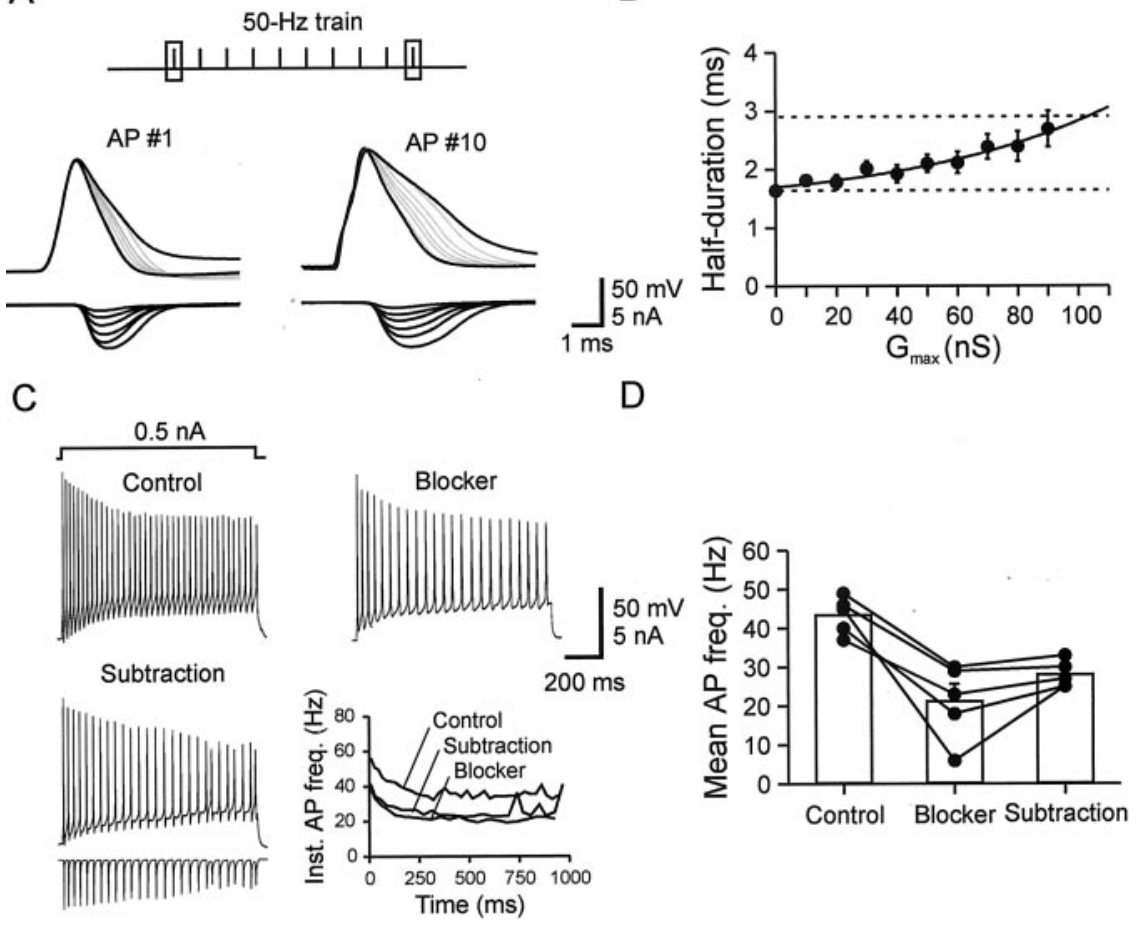

Figure 4. Mimicry of the effects of blockers by subtraction of Kv3 conductance with dynamic clamp. $A$, Single APs evoked by 0.5 msec pulses in an 0 A interneuron ( $3.5 \mathrm{nA}$; 1 st and 10 th AP in a $50 \mathrm{~Hz}$ train). $G_{\max }$ was varied from -20 to $-120 \mathrm{nS}$ in $-20 \mathrm{nS}$ steps. Note that AP broadening was induced by subtraction. For $G_{\max }=-120 \mathrm{nS}$, the effect of subtraction ( gray traces) mimics that of the pharmacological block of Kv3 channels (black traces, $0.3 \mathrm{~mm}$ 4-AP; longer AP). Note that the agreement is not perfect, which may be because of cell-to-cell variability in Kv3 channel gating or unspecific effects of the blockers. $B$, Half-duration of the 10th $A P$ in a $50 \mathrm{~Hz}$ train after subtraction of artificial conductance by dynamic clamp, plotted against $G_{\max }(n=10)$. The curve represents exponential function plus offset fitted to the data points. Dashed lines indicate the mean half-durations of the AP under control conditions (bottom line) and in the presence of blockers (top line). C, Trains of APs evoked by 1 sec pulses ( $0.5 \mathrm{nA}$ ). Traces were obtained under control conditions after subtraction of Kv3 conductance $\left(G_{\max }=-100 \mathrm{nS}\right)$ and in the presence of $1 \mathrm{~mm}$ TEA. The graph at the bottom right illustrates instantaneous AP frequency plotted against time for the three conditions. Data in $A$ and Care from different cells. D, Summary graph of mean AP frequency. Open bars indicate means; filled circles indicate single values for control, blocker (0.3 mм 4-AP or $1 \mathrm{~mm}$ TEA), and subtraction in the absence of blockers $(n=5)$.

deactivation rates failed to restore the FS phenotype in the same cells (Fig. 6B2,B3). In both cases, the mean AP frequency was reduced (from $40.6 \pm 1$ to $20.7 \pm 0.6$ and $22.6 \pm 2.1 \mathrm{~Hz}$, respectively; $n=8 ; p<0.05$ in both cases), although the underlying mechanisms appeared to be different. If the deactivation rate was slowed $\left(f_{\beta}=0.2\right)$, a low-frequency regular spiking pattern emerged (Fig. 6B2). In contrast, if the deactivation rate was accelerated $\left(f_{\beta}=5\right)$, the AP pattern became irregular (Fig. $6 \mathrm{~B} 3$ ), with periods of repetitive AP generation separated by silent or subthreshold oscillatory epochs (Kawaguchi, 1995). The difference in AP pattern between the two conditions was also reflected in the distribution of ISIs, which showed a small CV for $f_{\beta}=0.2$ (0.05) (Fig. 6B2, bottom) but a large CV for $f_{\beta}=5(0.42)$ (Fig. $6 B 3$, bottom).

To determine the mechanisms underlying the difference between $f_{\beta}=5$ and $f_{\beta}=0.2$, we compared maximal rates of rise $\left(d V / d t_{\max }\right)$, peak AP potentials, and peak fast afterhyperpolarization (fAHP) potentials between the two conditions (Fig. 6C). Adding artificial Kv3 conductance with accelerated deactivation $\left(f_{\beta}=5\right)$ led to a reduction in the maximal rate of rise, peak AP potential, and peak fAHP potential (Fig. $6 C$ ). Adding artificial $\mathrm{Kv} 3$ conductance with slowed deactivation $\left(f_{\beta}=0.2\right)$ had opposite effects (Fig. $6 C)(n=5)$. Thus, the mean AP frequency appeared to be reduced by the inactivation of voltage-gated $\mathrm{Na}^{+}$ channels in the first case $\left(f_{\beta}=5\right)$ and an increase in the fAHP amplitude in the second case $\left(f_{\beta}=0.2\right)$.

To obtain systematic information about the relationship between the mean AP frequency and $f_{\beta}$, we varied $f_{\beta}$ and stimulus intensity $(I)$ over a wide range $\left(f_{\beta}=0.1-10 ; I=0.1-0.7 \mathrm{nA}\right)$. Figure $6 \mathrm{D}$ shows the mean $\mathrm{AP}$ frequency during the 1 sec pulse, plotted against $f_{\beta}$ and $I$. For a given stimulus current, the relationship did not increase monotonically but rather showed a relatively sharp maximum corresponding to $f_{\beta} \approx 1.2$. In summary, these results indicate that the deactivation kinetics of Kv3 channels are optimized to produce FS with near-maximal AP frequency in $\mathrm{OA}$ interneurons.

\section{Lowering the activation threshold converts the AP phenotype from FS to adapting}

The high activation threshold is another hallmark of Kv3 channels (Rudy and McBain, 2001). To examine the impact of this gating property on the FS phenotype, we added artificial Kv3 channels with altered $V_{1 / 2}$ using the dynamic-clamp system (Fig. 7). A negative shift of $V_{1 / 2}$ led to a conversion from an FS to an adapting phenotype. If $V_{1 / 2}$ was shifted by $-20 \mathrm{mV}$, the threshold for the initiation of multiple APs was increased, but the ability to generate high-frequency spike trains was preserved (Fig. 7B2). If $V_{1 / 2}$ was shifted by $-30 \mathrm{mV}$, even during high stimuli, only a single AP (three of seven cells) or a small number of APs (four of seven cells) were evoked (Fig. $7 B 3$ ). Figure $7 C$ shows the mean AP frequency during the $1 \mathrm{sec}$ pulse, plotted against the shift of the midpoint potential $(\Delta V)$ and the injected current. A shift toward negative potentials changed the frequency-current curves from a hyperbolic to a sigmoidal shape, indicating that the input-output relationship of the neuron is dependent on the $\mathrm{K}^{+}$channel activation curve. In conclusion, these results show that the high activation threshold of $\mathrm{Kv} 3$ channels facilitates FS. In contrast, an artificially generated low activation threshold confers adaptation of AP frequency during a long stimulus.

\section{Absence of fast inactivation of Kv3 ensures constancy of AP duration, whereas presence of inactivation leads to activity- dependent broadening}

The absence of fast inactivation distinguishes Kv3.1 and Kv3.2, expressed in OA interneurons, from Kv3.3 and Kv3.4 (Rudy and McBain, 2001). To examine how the absence versus the presence of inactivation influences the AP pattern, we introduced an artificial inactivation process in our dynamic-clamp system (Fig. 8). Original and altered Kv3 channels were equally effective in restoring the duration of the first AP evoked by high-frequency trains of stimuli. However, unlike the original Kv3 channel, the altered $\mathrm{Kv} 3$ channel failed to rescue the duration of the 10th AP (Fig. $8 \mathrm{~B}, \mathrm{C}$ ). After the addition of the original $\mathrm{Kv} 3$ conductance, the ratio of half-durations of the 10th to the first AP was $1.22 \pm$ 


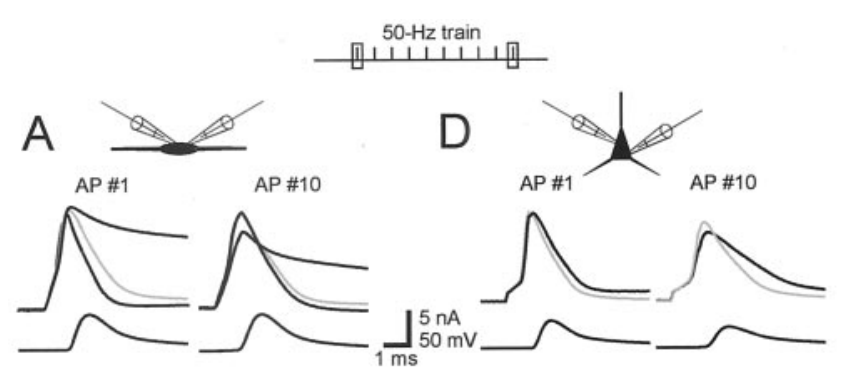

B
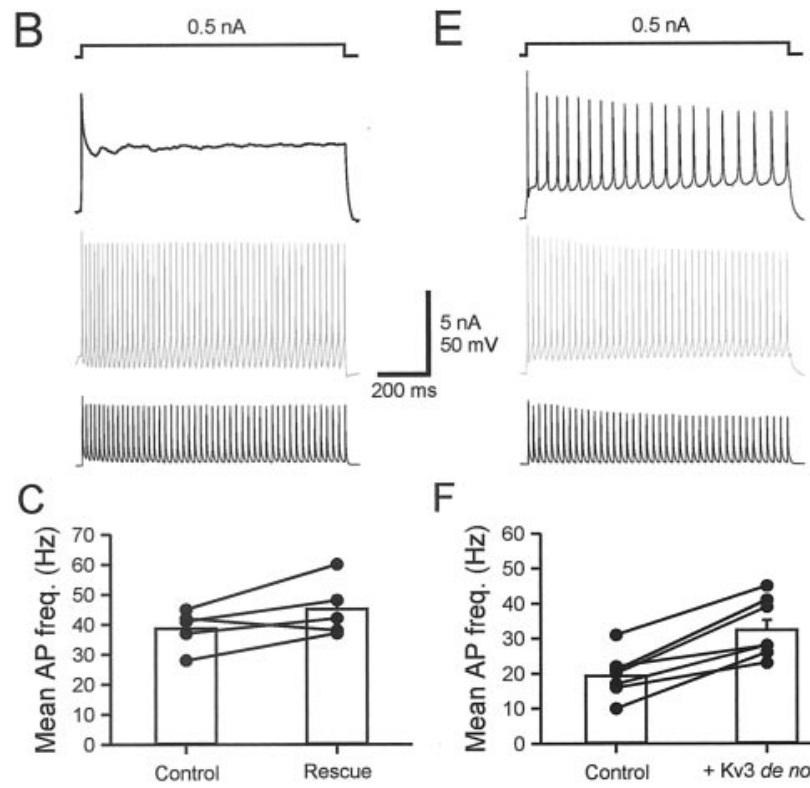

$\coprod_{200 \mathrm{~ms}}^{5 \mathrm{nA}} 50 \mathrm{mV}$
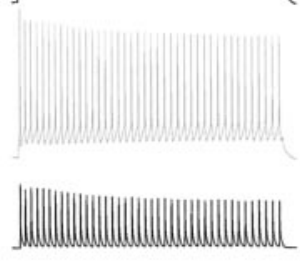

$\mathrm{F}$

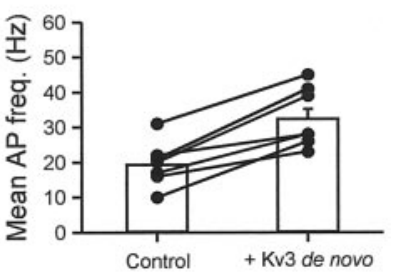

Figure 5. Kv3 conductance is sufficient to induce the FS phenotype in two different host-cell types. $A-C$, Induction of FS phenotype in $0 A$ interneurons after complete pharmacological block of voltage-gated $\mathrm{K}^{+}$channels. Traces were obtained in the presence of $5 \mathrm{~mm} 4-\mathrm{AP}$ plus $20 \mathrm{~mm}$ TEA and after the addition of artificial Kv3 conductance in the presence of blockers. A, Single APs evoked by 0.5 msec pulses ( $3.5 \mathrm{nA}$; 1 st and 10 th $A P$ in a $50 \mathrm{~Hz}$ train). $B$, APs evoked by 1 sec pulses $(0.5 \mathrm{nA})$. Data in $A$ and $B$ are from the same cell $\left(G_{\max }=100 \mathrm{nS}\right)$. C, Summary graph of mean AP frequency. Open bars indicate means; filled circles indicate single values for control and rescue by artificial Kv3 conductance $(n=5)$. Note that the AP frequency was increased beyond the control value by the artificial Kv3 conductance in four of five cells ("over-rescue"), which may be attributable to the anti-FS effects of voltage-gated $\mathrm{K}^{+}$channels other than Kv3.D-F, De novo induction of FS phenotype in pyramidal neurons. D, Single APs evoked by $0.5 \mathrm{msec}$ pulses ( $3.5 \mathrm{nA}$; $1 \mathrm{st}$ and 10th $A P$ in a $50 \mathrm{~Hz}$ train) in a (A1 pyramidal neuron. No blockers were applied. $E$, Trains of APs evoked by 1 sec pulses $(0.5 \mathrm{nA}, \sim 5$ times threshold). Traces were obtained under control conditions and after the addition of artificial Kv 3 conductance. Data in $D$ and $E$ are from the same cell $\left(G_{\max }=100 \mathrm{nS}\right)$.F, Summary graph of mean AP frequency. Open bars indicate means; filled circles indicate single values for controls and after the addition of artificial Kv3 conductance in the presence of blockers $(n=8)$.

0.03 for a $50 \mathrm{~Hz}$ train and $1.44 \pm 0.06$ for a $70 \mathrm{~Hz}$ train, implying approximate constancy of AP duration. In contrast, after the addition of the inactivating Kv3 conductance, the spike duration ratio measured in the same cells using identical paradigms was markedly larger $(1.83 \pm 0.10$ and $2.1 \pm 0.1$, respectively; $n=4$; $p<0.05$ ) (Fig. 8C). Thus the original Kv3 channel confers constancy to AP duration, whereas an inactivating Kv3 conductance mediates AP broadening.

Finally, we examined the impact of the absence versus the presence of inactivation on the FS phenotype. Compared with the original Kv3 channel, the inactivating channel was significantly less effective in restoring FS (Fig. 8D,E). After addition of the inactivating $\mathrm{Kv} 3$ channels, the mean AP frequency during long current pulses was $29.0 \pm 1.5 \mathrm{~Hz}$, significantly smaller than that after the addition of the original Kv3 channels in the same cells $(43.4 \pm 1.6 \mathrm{~Hz} ; n=11 ; p<0.005)$ (Fig. $8 \mathrm{E}$ ). In conclusion, these results indicate that the absence of fast inactivation of the Kv3 channel subtypes expressed in OA interneurons is important for the FS phenotype.

\section{Discussion}

Kv3 channels are necessary, and perhaps sufficient, for the FS phenotype

To examine the relationship between the AP phenotype and the functional properties of $\mathrm{Kv} 3$ channels, we chose the OA interneuron in the hippocampal CA1 region as a model. First, the $\mathrm{K}^{+}$ channels expressed in this cell type are characterized functionally and molecularly (Zhang and McBain, 1995a,b; Martina et al., 2000; Lien et al., 2002). Second, the site of AP initiation and the sequence of AP propagation in this neuron are well defined (Martina et al., 2000). Finally, the elongated shape of the soma and proximal dendrites allowed us to perform two electrode wholecell current-clamp recordings (Martina et al., 2000), which avoids problems caused by a voltage drop across the series resistance. However, the approach reported here is versatile and can be applied to any type of cellular or subcellular element that is sufficiently large to accommodate two patch electrodes.

We used the dynamic-clamp approach, which allowed us to delete, rescue, and add altered voltage-gated conductance in a defined manner. This experimental design is much more flexible than that of pharmacological (Martina et al., 1998; Erisir et al., 1999), antisense (Vincent et al., 2000), and knock-out or transgenic experiments (Ho et al., 1997; Lau et al., 2000; Espinosa et al., 2001). A potential limitation of the dynamic-clamp technique is that the artificial conductance is generated at a somatic point source. However, the validity of our approach is supported by two major arguments. First, the addition of artificial Kv3 after pharmacological block reverses the effects of the blocker, whereas subtraction mimics it. In both cases, the $G_{\max }$ values are comparable with perisomatic conductances estimated from nucleated patch recordings. This is unlikely to occur if artificial and real channels are very far apart. Second, immunocytochemical analysis independently indicates that Kv3 channels in interneurons are located perisomatically (Weiser et al., 1995; Chow et al., 1999; Ozaita et al., 2002; Tansey et al., 2002).

Both the rescue of the FS phenotype in OA interneurons after complete pharmacological block of voltage-gated $\mathrm{K}^{+}$channels and the de novo induction of the FS phenotype in regularly spiking CA1 pyramidal neurons provide evidence that Kv3 channels are necessary for the FS phenotype and can be sufficient, at least under the conditions used here (Figs. 3, 5). Thus, Kv3 channels appear to be the main determinant of FS. The observation that FS can be induced in both OA interneurons and CA1 pyramidal cells (Fig. 5) also shows that Kv3 channel expression may be a more important factor for shaping the AP phenotype than somatodendritic morphology, which is very different between the two cell types (Mainen and Sejnowski, 1996).

\section{Impact of individual gating properties of $\mathrm{Kv} 3$}

The dynamic-clamp approach allowed us to examine the impact of conductance and individual gating properties of Kv3 channels, such as the deactivation rate. This is not possible in real channels because kinetic and steady-state parameters are tightly linked (Shieh et al., 1997). The major emerging rules are as follows: (1) The deactivation rate is a major determinant of AP frequency and pattern (Fig. 6). However, the relationship between the deactivation rate and mean AP frequency during a long current pulse is 

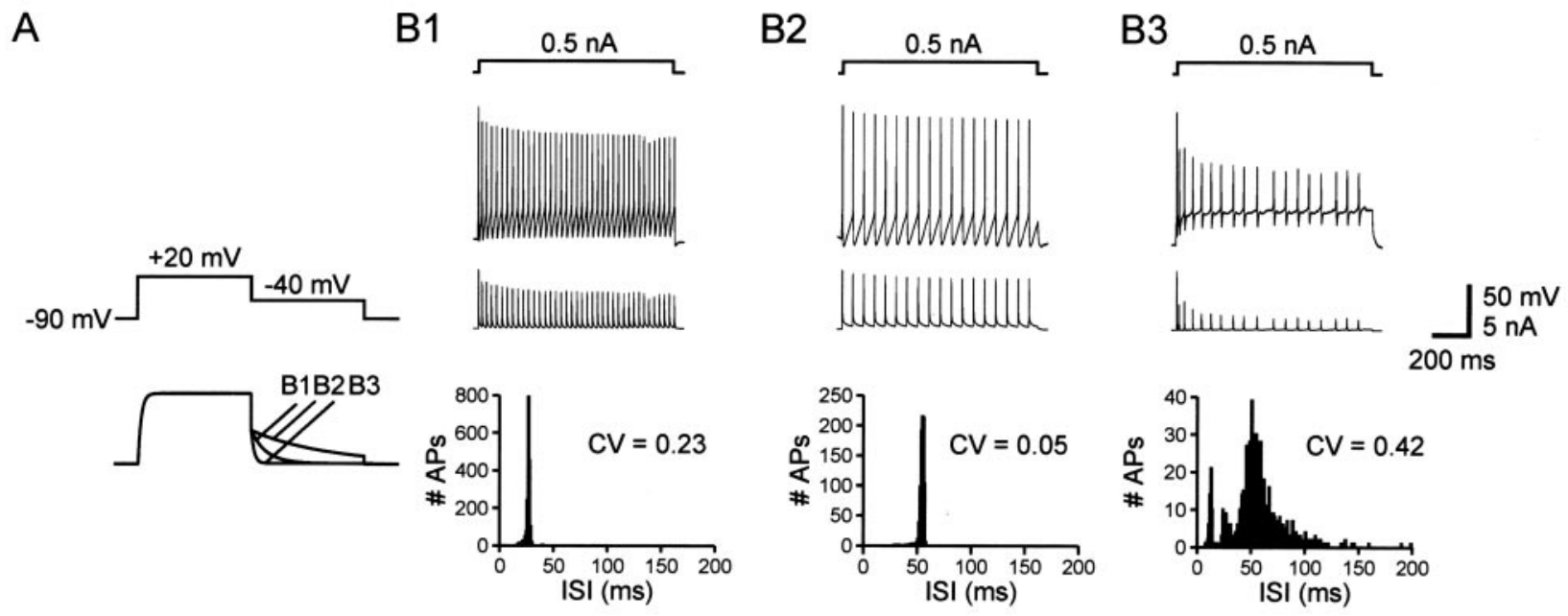

C1

C2

\section{C3}
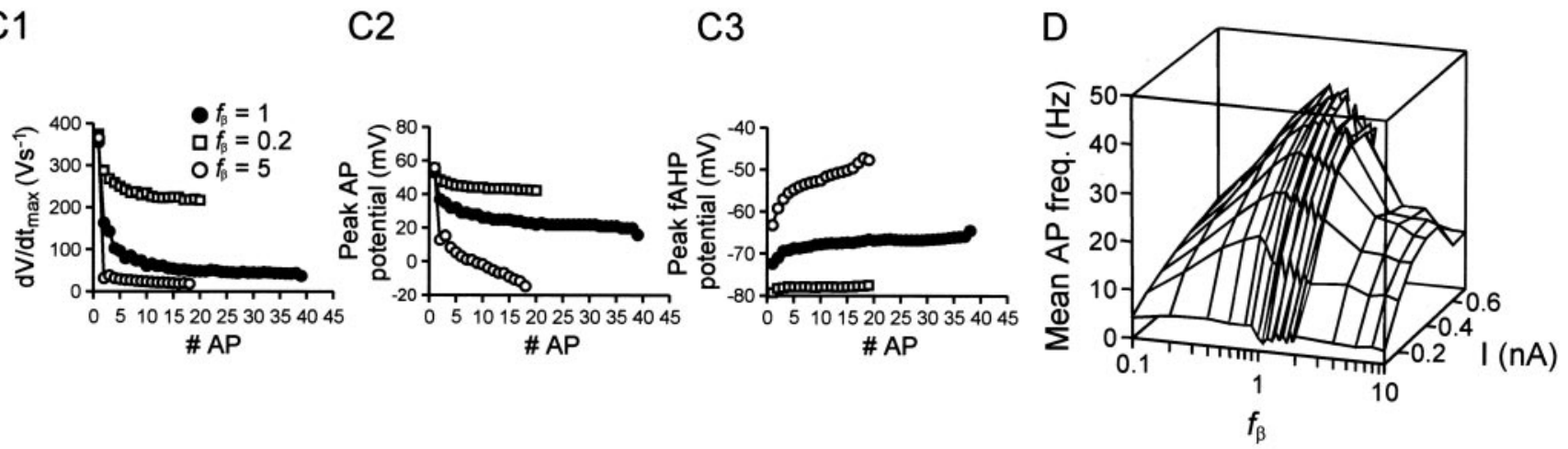

Figure 6. Optimal deactivation kinetics for FS in 0 A interneurons. $A$, Schematic illustration of the selective change in deactivation kinetics. $B$, Trains of APs evoked by 1 sec pulses ( $0.5 \mathrm{nA})$. B1, Traces after the addition of artificial Kv3 conductance (deactivation rate unchanged; i.e., deactivation factor $f_{\beta}=1$ ) by dynamic clamp in the presence of $0.3 \mathrm{~mm} 4$ 4-AP. The graphs at the bottom are histograms of ISIs from 50 sweeps (including B1) recorded from the same cell. B2, Data after the addition of Kv3 channels with a reduced deactivation rate $\left(f_{\beta}=0.2\right) . B 3$, Data after the addition of $\mathrm{Kv} 3$ channels with an increased deactivation rate $\left(f_{\beta}=5\right)$. Note that the $\mathrm{CV}$ of the ISI is much larger in $B 3$ than in $B 2$, suggesting that the mean $A P$ frequency is reduced by different mechanisms. Data in $B 1-B 3$ are from the same cell $\left(G_{\max }=140 \mathrm{nS}\right)$. The peak dynamic-clamp current during the first AP is similar in the three conditions $(5.31 \pm 0.05,5.42 \pm 0.01$, and 5.49 $\pm 0.02 \mathrm{nA}) . C$, Analysis of the mechanisms underlying the reduction of mean AP frequency. (1, Maximal rate of rise of the AP. C2, Peak AP potential. C 3 , Peak fAHP potential. Filled circles, $f_{\beta}=1 ;$ open squares, $f_{\beta}=$ 0.2; open circles, $f_{\beta}=5$. Lines connect data points for a single condition. D, A three-dimensional representation of mean AP frequency during the 1 sec pulse against $f_{\beta}$ and the amplitude of the current pulse. Every line crossing represents a mean frequency value $(n=3-7)$. Note that the maximal AP frequency is reached at $f_{\beta} \approx 1.2$, corresponding to a deactivation rate slightly above the original value.

not simple. The mean AP frequency does not increase monotonically with the deactivation rate, as might be expected, but rather shows a maximal value at an intermediate rate (Fig. 6D). This optimum relationship appears to emerge from the interaction between Kv3 channels and the recovery of voltage-gated $\mathrm{Na}^{+}$ channels from inactivation. If deactivation is too fast, the AP frequency is slowed because the fAHP becomes very brief, and the recovery of $\mathrm{Na}^{+}$channels from inactivation is insufficient (Kuo and Bean, 1994; Martina and Jonas, 1997; Erisir et al., 1999). This converts the AP pattern from FS to irregular spiking. In contrast, if deactivation is too slow, AP frequency is reduced because the fAHP is prolonged, and the threshold for the next spike is reached later. This switches the AP pattern from FS to slow regular spiking. In $\mathrm{OA}$ interneurons, the deactivation rate producing the maximal mean firing rate is slightly higher than the natural value $\left(f_{\beta} \approx 1.2\right)$, suggesting room for acceleration of the spiking rate. (2) The high activation threshold also facilitates FS (Fig. 7). If the activation curve is shifted to more negative voltages, FS is impaired and gradually replaced by adaptation. For a shift of -30 $\mathrm{mV}$, only a single spike is generated during a long depolarizing current pulse (Fig. 7B3). (3) Finally, the absence of fast inactivation facilitates FS (Fig. 8). If inactivation is included in the Kv3 model, the AP frequency is reduced. Concomitantly marked AP broadening is induced, at least in the rather extreme form of inactivation implemented here (100\% extent, slow recovery).

Physiological changes of Kv3 channel gating and explanation of natural variants of AP patterns of interneurons

The changes in Kv3 channel gating implemented in the dynamicclamp system may also occur under physiological or pathophysiological conditions, for example via regulation of Kv subunit expression (Martina et al., 1998) or through the action of neuromodulators (Levitan, 1994). Changes in the deactivation time constant could be generated by differential expression of Kv3.1 and Kv3.2, which have deactivation rates differing by a factor of $\approx 2$ (Hernández-Pineda et al., 1999). Alterations in midpoint potential may be caused by dephosphorylation via inhibition of casein kinase activity, which shifts the activation curve of recombinant and native Kv3 channels by $-20 \mathrm{mV}$ (Macica and Kaczmarek, 2001); comparable effects on Kv4-like channels are discussed by Hoffman and Johnston (1998). This change may be sufficient to switch an FS into an adapting phenotype. Finally, the extent and time course of inactivation could be regulated by differential expression of Kv3.1-Kv3.2 and Kv3.3-Kv3.4 (the inac- 
A
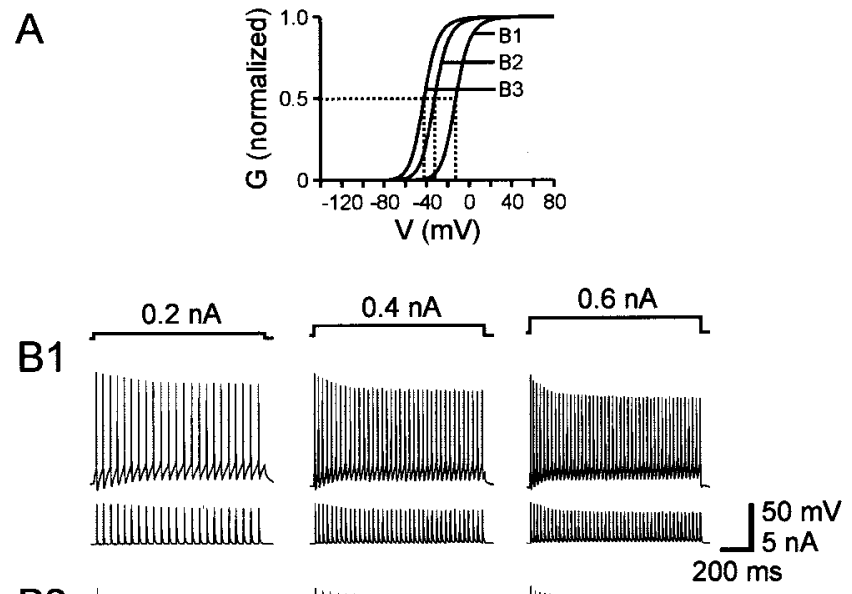

B2
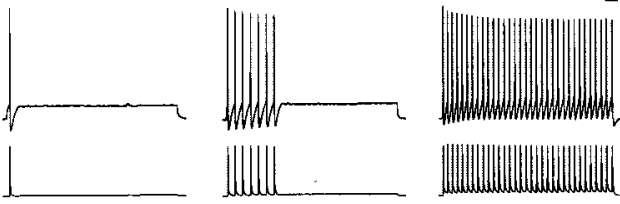

B3

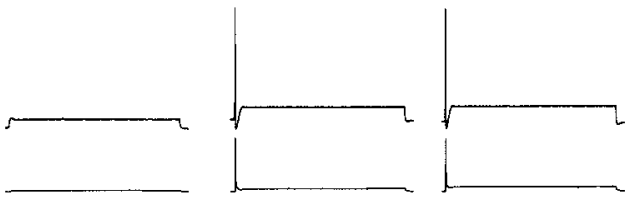

C

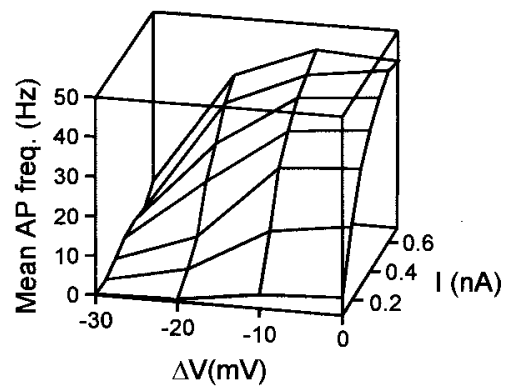

Figure 7. A high activation threshold facilitates $F S$, whereas a low activation threshold leads to adaptation. $A$, Schematic illustration of the change in midpoint potential $V_{1 / 2}$ of the activation curve. Dashed lines indicate midpoint potentials. $B$, Trains of APs evoked by 1 sec pulses (stimulus current of $0.2,0.4$, and $0.6 \mathrm{nA}$ ). Traces were obtained after the addition of artificial Kv3 conductance in the presence of $1 \mathrm{~mm}$ TEA. B1, Data for the addition of original Kv3. B2, B3, Data for the addition of Kv3 after the shift of $V_{1 / 2}$ by $-20 \mathrm{mV}$ (B2) or $-30 \mathrm{mV}$ (B3). Note the strong adaptation induced by the shift of $V_{1 / 2}$. Data in $B 1-B 3$ are from the same cell $\left(G_{\max }=110 \mathrm{nS}\right)$. $C, A$ three-dimensional representation of mean AP frequency during the $1 \mathrm{sec}$ pulse against the change in $V_{1 / 2}$ and the amplitude of the current pulse. Every line crossing represents a mean frequency value $(n=7)$. Note that a shift to more negative potentials reduces the mean AP frequency by promoting adaptation.

tivating members of the Kv3 family) (Rudy and McBain, 2001) or by changes in the intracellular redox status of interneurons (Ruppersberg et al., 1991).

Conversely, the results of the dynamic-clamp experiments explain several naturally occurring AP patterns. Intermittent or "stuttering" firing is occasionally observed in FS GABAergic interneurons in different brain regions during low-intensity stimuli (Kawaguchi, 1995). In addition, irregular spiking after a highfrequency burst of APs is found in VIP-positive neocortical GABAergic interneurons (Cauli et al., 1997). Our results suggest that the expression of $\mathrm{K}^{+}$channels with accelerated deactivation could generate such AP phenotypes (Fig. 6 B3). Single spike or "damped" AP phenotypes are found abundantly in neurons of the auditory pathway (e.g., in the medial nucleus of the trapezoid

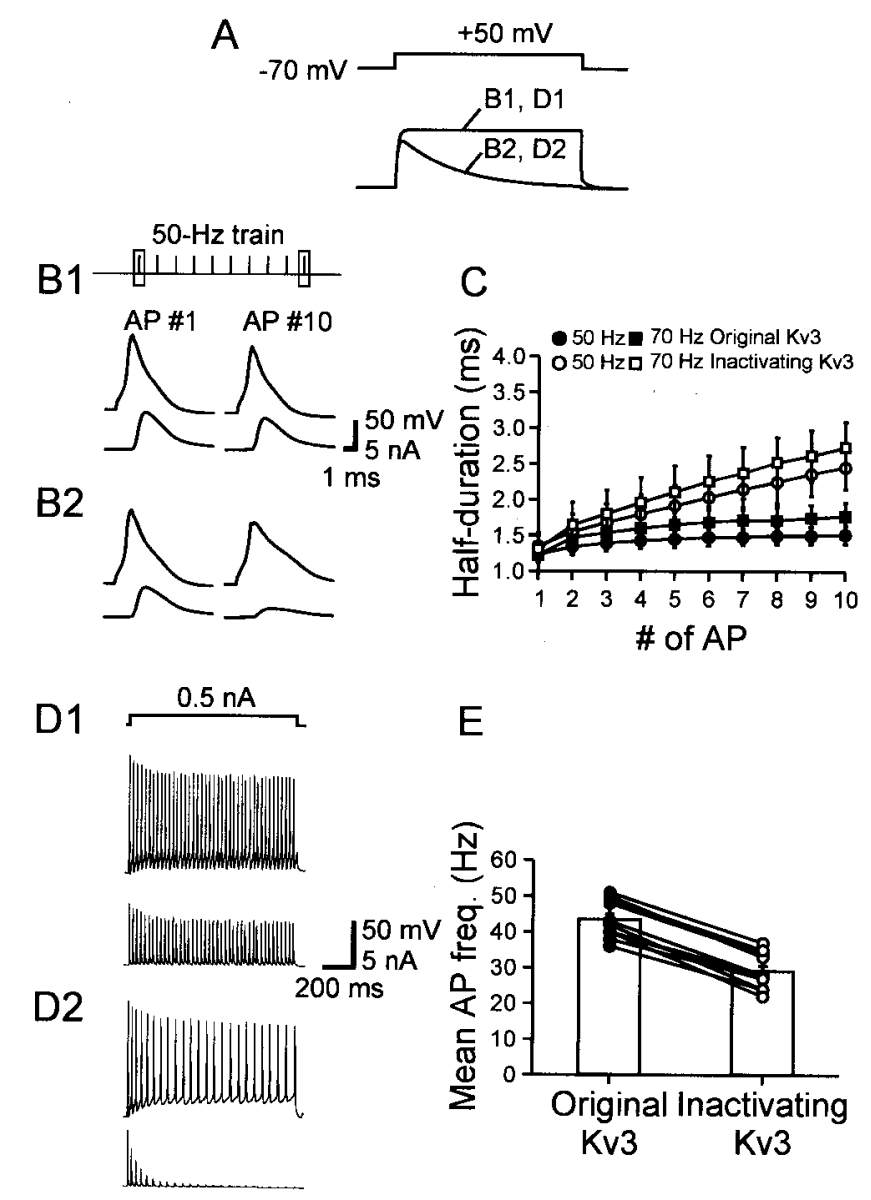

Figure 8. Noninactivating channels promote FS, whereas inactivating channels lead to activity-dependent AP broadening. $A$, Schematic illustration of the change in inactivation. $B$, Single APs evoked by $0.5 \mathrm{msec}$ pulses ( $3.5 \mathrm{nA}$; 1st and 10th AP in a $50 \mathrm{~Hz}$ train). Traces were obtained in the presence of $1 \mathrm{~mm}$ TEA after the addition of either original Kv3 channels $(B 1)$ or inactivating Kv3 channels (B2). C, Half-duration of APs in a high-frequency train in the presence of $1 \mathrm{~mm}$ TEA after the addition of artificial Kv3 conductance. Filled symbols, original Kv3; open symbols, inactivating Kv3 channels $(n=4)$. Circles indicate $50 \mathrm{~Hz}$ stimulation; squares indicate $70 \mathrm{~Hz}$ stimulation. Lines connect data points for a single condition. D, Trains of APs evoked by 1 sec pulses $(0.5 \mathrm{nA})$. Traces were obtained in the presence of $1 \mathrm{~mm}$ TEA after the addition of either original Kv3 channels (D1) or inactivating Kv3 channels (D2). Note that the AP-related dynamicclamp current decreases substantially in $B 2$ and $D 2$ during the train of APs, indicating cumulative inactivation. Data in $B$ and $D$ were obtained from the same cell $\left(G_{\max }=160 \mathrm{nS}\right)$. E, Summary graph of mean AP frequency. Open bars indicate means; filled circles indicate single values for original Kv3; open circles indicate inactivating Kv3 $(n=11)$.

body) (Brew and Forsythe, 1995; Trussell, 1997; Soares et al., 2002). Our results suggest that the expression of $\mathrm{K}^{+}$channels with a negative activation curve may generate such AP patterns (Fig. 7B3). Consistent with this hypothesis, the block of lowthreshold $\mathrm{K}^{+}$channels (presumably Kv1) by dendrotoxin abolishes adaptation (Brew and Forsythe, 1995; Wang et al., 1998; Bekkers and Delaney, 2001). Finally, activity-dependent AP broadening was reported in molluscan neurons (Aldrich et al., 1979; Ma and Koester, 1995; Whim and Kaczmarek, 1998), hypophyseal nerve terminals (Jackson et al., 1991), and hippocampal mossy fiber boutons (Geiger and Jonas, 2000). In our experiments, AP broadening is mimicked when an artificially inactivating Kv3 channel is applied in dynamic-clamp experiments.

Our results suggest a general picture of how characteristically diverse AP patterns of central neurons are generated in the mammalian brain. Kv3-like channels with fast deactivation, high acti- 
vation threshold, and lack of inactivation promote FS. In addition, both low-threshold $\mathrm{K}^{+}$channels (Kv1-like) and inactivating $\mathrm{K}^{+}$channels (e.g., Kv4-like) suppress FS and promote adaptation. Similarly, previous functional and molecular studies suggested that the balance between the expression of Kv3 channels and slowly activating and deactivating Kv2 channels determines the ability to discharge at high rates (Baranauskas et al., 1999). Collectively, these results suggest that the balance between the conductance density of $\mathrm{Kv} 3$ channels and that of the other voltage-gated $\mathrm{K}^{+}$channels (Kv1, Kv2, and Kv4) controls the firing pattern of interneurons and principal cells in the CNS.

\section{Functional significance for synaptic inhibition and network activity}

Throughout the CNS, Kv3 channels are expressed not only in somata but also in presynaptic terminals (Moreno et al., 1995; Weiser et al., 1995; Rudy and McBain, 2001; Ozaita et al., 2002). Thus, our results may have implications for the dynamics of GABA release from interneurons. Presynaptic Kv3 channels will keep the presynaptic AP brief, which may ensure a high synchrony of vesicular GABA release (Kraushaar and Jonas, 2000). Additionally, Kv3 channels may prevent AP broadening during AP trains. This could be a key mechanism underlying releaseindependent components of paired-and multiple-pulse depression of inhibitory synaptic transmission (Kraushaar and Jonas, 2000; Maccaferri et al., 2000; Hefft et al., 2002). Finally, axonal Kv3 channels may ensure both reliability and temporal fidelity of conduction from the initiation site to the presynaptic terminals during high-frequency trains (Debanne et al., 1997).

The quantitative relationship between Kv3 gating and fast spiking addressed here also has implications for neuronal network function. Interneuron networks are thought to be involved in the generation of network oscillations in the gamma (30-90 $\mathrm{Hz}$ ) and ripple frequency range $(>100 \mathrm{~Hz})$ (Wang and Buzsáki, 1996; Bartos et al., 2001). During this oscillatory activity, FS interneurons fire at frequencies of up to several hundred Hertz, with APs phase-locked to the oscillation cycles in the field potential (Ylinen et al., 1995; Jones et al., 2000). Evidently, the expression of $\mathrm{Kv} 3$ channels is a key requirement for the participation of interneurons in this high-frequency activity. The important contribution of $\mathrm{Kv} 3$ channels to high-frequency oscillatory activity in interneurons is also supported by interneuron network simulations, which show that Kv3 deactivation kinetics has a marked influence on the frequency but not on the coherence of network oscillations (Bartos et al., 2001; P. Jonas, unpublished data). Transgenic mice in which gating of Kv3 is modified may allow us to test this hypothesis directly in vivo.

In conclusion, our results show that $\mathrm{Kv} 3$ channels are necessary for FS and can even be sufficient under certain conditions. They demonstrate that fast deactivation kinetics, high activation threshold, and lack of inactivation of Kv3 are essential for highfrequency repetitive activity. Conversely, they provide direct evidence for causal links between low activation threshold and adaptation as well as between inactivation and AP broadening. Thus, our results contribute to our understanding of the complexity of AP patterns in central neurons.

\section{References}

Aldrich RW, Getting PA, Thompson SH (1979) Mechanism of frequencydependent broadening of molluscan neurone soma spikes. J Physiol (Lond) 291:531-544.

Atzori M, Lau D, Tansey EP, Chow A, Ozaita A, Rudy B, McBain CJ (2000) $\mathrm{H}_{2}$ histamine receptor-phosphorylation of Kv3.2 modulates interneuron fast spiking. Nat Neurosci 3:791-798.
Baranauskas G, Tkatch T, Surmeier DJ (1999) Delayed rectifier currents in rat globus pallidus neurons are attributable to Kv2.1 and $\mathrm{Kv} 3.1 / 3.2 \mathrm{~K}^{+}$ channels. J Neurosci 19:6394-6404.

Bartos M, Vida I, Frotscher M, Geiger JRP, Jonas P (2001) Rapid signaling at inhibitory synapses in a dentate gyrus interneuron network. J Neurosci 21:2687-2698.

Bekkers JM, Delaney AJ (2001) Modulation of excitability by $\alpha$-dendrotoxinsensitive potassium channels in neocortical pyramidal neurons. J Neurosci 21:6553-6560.

Betancourt L, Colom LV (2000) Potassium $\left(\mathrm{K}^{+}\right)$channel expression in basal forebrain cholinergic neurons. J Neurosci Res 61:646-651.

Brew HM, Forsythe ID (1995) Two voltage-dependent $\mathrm{K}^{+}$conductances with complementary functions in postsynaptic integration at a central auditory synapse. J Neurosci 15:8011-8022.

Cauli B, Audinat E, Lambolez B, Angulo MC, Ropert N, Tsuzuki K, Hestrin S, Rossier J (1997) Molecular and physiological diversity of cortical nonpyramidal cells. J Neurosci 17:3894-3906.

Chow A, Erisir A, Farb C, Nadal MS, Ozaita A, Lau D, Welker E, Rudy B (1999) $\mathrm{K}^{+}$channel expression distinguishes subpopulations of parvalbumin- and somatostatin-containing neocortical interneurons. J Neurosci 19:9332-9345.

Connors BW, Gutnick MJ (1990) Intrinsic firing patterns of diverse neocortical neurons. Trends Neurosci 13:99-104.

Debanne D, Guérineau NC, Gähwiler BH, Thompson SM (1997) Actionpotential propagation gated by an axonal $\mathrm{I}_{\mathrm{A}}$-like $\mathrm{K}^{+}$conductance in hippocampus. Nature 389:286-289.

Du J, Zhang L, Weiser M, Rudy B, McBain CJ (1996) Developmental expression and functional characterization of the potassium-channel subunit Kv3.1b in parvalbumin-containing interneurons of the rat hippocampus. J Neurosci 16:506-518.

Erisir A, Lau D, Rudy B, Leonard CS (1999) Function of specific $\mathrm{K}^{+}$channels in sustained high-frequency firing of fast-spiking neocortical interneurons. J Neurophysiol 82:2476-2489.

Espinosa F, McMahon A, Chan E, Wang S, Ho CS, Heintz N, Joho RH (2001) Alcohol hypersensitivity, increased locomotion, and spontaneous myoclonus in mice lacking the potassium channels Kv3.1 and Kv3.3. J Neurosci 21:6657-6665.

Foster WR, Ungar LH, Schwaber JS (1993) Significance of conductances in Hodgkin-Huxley models. J Neurophysiol 70:2502-2518.

Geiger JRP, Jonas P (2000) Dynamic control of presynaptic $\mathrm{Ca}^{2+}$ inflow by fast-inactivating $\mathrm{K}^{+}$channels in hippocampal mossy fiber boutons. Neuron 28:927-939.

Goldman MS, Golowasch J, Marder E, Abbott LF (2001) Global structure, robustness, and modulation of neuronal models. J Neurosci 21:5229-5238.

Hefft S, Kraushaar U, Geiger JRP, Jonas P (2002) Presynaptic short-term depression is maintained during regulation of transmitter release at a GABAergic synapse in rat hippocampus. J Physiol (Lond) 539:201-208.

Hernández-Pineda R, Chow A, Amarillo Y, Moreno H, Saganich M, VegaSaenz de Miera E, Hernández-Cruz A, Rudy B (1999) Kv3.1-Kv3.2 channels underlie a high-voltage-activating component of the delayed rectifier $\mathrm{K}^{+}$current in projecting neurons from the globus pallidus. J Neurophysiol 82:1512-1528.

Hille B (2001) Ion channels of excitable membranes, Ed 3. Sunderland, MA: Sinauer.

Ho CS, Grange RW, Joho RH (1997) Pleiotropic effects of a disrupted K ${ }^{+}$ channel gene: reduced body weight, impaired motor skill and muscle contraction, but no seizures. Proc Natl Acad Sci USA 94:1533-1538.

Hodgkin AL, Huxley AF (1952) A quantitative description of membrane current and its application to conduction and excitation in nerve. J Physiol (Lond) 117:500-544.

Hoffman DA, Johnston D (1998) Downregulation of transient $\mathrm{K}^{+}$channels in dendrites of hippocampal CA1 pyramidal neurons by activation of PKA and PKC. J Neurosci 18:3521-3528.

Jackson MB, Konnerth A, Augustine GJ (1991) Action potential broadening and frequency-dependent facilitation of calcium signals in pituitary nerve terminals. Proc Natl Acad Sci USA 88:380-384.

Jan LY, Jan YN (1997) Cloned potassium channels from eukaryotes and prokaryotes. Annu Rev Neurosci 20:91-123.

Jones MS, MacDonald KD, Choi B, Dudek FE, Barth DS (2000) Intracellular correlates of fast $(>200 \mathrm{~Hz})$ electrical oscillations in rat somatosensory cortex. J Neurophysiol 84:1505-1518.

Kawaguchi Y (1995) Physiological subgroups of nonpyramidal cells with 
specific morphological characteristics in layer II/III of rat frontal cortex. J Neurosci 15:2638-2655.

Kraushaar U, Jonas P (2000) Efficacy and stability of quantal GABA release at a hippocampal interneuron-principal neuron synapse. J Neurosci 20:5594-5607.

Kuo C-C, Bean BP (1994) $\mathrm{Na}^{+}$channels must deactivate to recover from inactivation. Neuron 12:819-829.

Lau D, Vega-Saenz de Miera E, Contreras D, Ozaita A, Harvey M, Chow A, Noebels JL, Paylor R, Morgan JI, Leonard CS, Rudy B (2000) Impaired fast-spiking, suppressed cortical inhibition, and increased susceptibility to seizures in mice lacking $\mathrm{Kv} 3.2 \mathrm{~K}^{+}$channel proteins. J Neurosci 20:9071-9085.

Levitan IB (1994) Modulation of ion channels by protein phosphorylation and dephosphorylation. Annu Rev Physiol 56:193-212.

Lien CC, Jonas P (2002) How do Kv3-like $\mathrm{K}^{+}$channels in hippocampal interneurons facilitate fast spiking? Soc Neurosci Abstr 28:546.12.

Lien C-C, Martina M, Schultz JH, Ehmke H, Jonas P (2002) Gating, modulation and subunit composition of voltage-gated $\mathrm{K}^{+}$channels in dendritic inhibitory interneurones of rat hippocampus. J Physiol (Lond) 538:405-419.

Ma M, Koester J (1995) Consequences and mechanisms of spike broadening of R20 cells in Aplysia californica. J Neurosci 15:6720-6734.

Ma M, Koester J (1996) The role of $\mathrm{K}^{+}$currents in frequency-dependent spike broadening in Aplysia R20 neurons: a dynamic-clamp analysis. J Neurosci 16:4089-4101.

Maccaferri G, Roberts JDB, Szucs P, Cottingham CA, Somogyi P (2000) Cell surface domain specific postsynaptic currents evoked by identified GABAergic neurones in rat hippocampus in vitro. J Physiol (Lond) 524:91-116.

Macica CM, Kaczmarek LK (2001) Casein kinase 2 determines the voltage dependence of the Kv3.1 channel in auditory neurons and transfected cells. J Neurosci 21:1160-1168.

Magistretti J, Mantegazza M, de Curtis M, Wanke E (1998) Modalities of distortion of physiological voltage signals by patch-clamp amplifiers: a modeling study. Biophys J 74:831-842.

Mainen ZF, Sejnowski TJ (1996) Influence of dendritic structure on firing pattern in model neocortical neurons. Nature 382:363-366.

Martina M, Jonas P (1997) Functional differences in $\mathrm{Na}^{+}$channel gating between fast-spiking interneurones and principal neurones of rat hippocampus. J Physiol (Lond) 505:593-603.

Martina M, Schultz JH, Ehmke H, Monyer H, Jonas P (1998) Functional and molecular differences between voltage-gated $\mathrm{K}^{+}$channels of fastspiking interneurons and pyramidal neurons of rat hippocampus. J Neurosci 18:8111-8125.

Martina M, Vida I, Jonas P (2000) Distal initiation and active propagation of action potentials in interneuron dendrites. Science 287:295-300.

McBain CJ, Fisahn A (2001) Interneurons unbound. Nat Rev Neurosci 2:11-23.

Moreno H, Kentros C, Bueno E, Weiser M, Hernandez A, Vega-Saenz de Miera E, Ponce A, Thornhill W, Rudy B (1995) Thalamocortical projections have a $\mathrm{K}^{+}$channel that is phosphorylated and modulated by cAMPdependent protein kinase. J Neurosci 15:5486-5501.

Ozaita A, Martone ME, Ellisman MH, Rudy B (2002) Differential subcellular localization of the two alternatively spliced isoforms of the Kv3.1 potassium channel subunit in brain. J Neurophysiol 88:394-408.

Porcello DM, Ho CS, Joho RH, Huguenard JR (2002) Resilient RTN fast spiking in Kv3.1 null mice suggests redundancy in the action potential repolarization mechanism. J Neurophysiol 87:1303-1310.

Rettig J, Wunder F, Stocker M, Lichtinghagen R, Mastiaux F, Beckh S, Kues W, Pedarzani P, Schröter KH, Ruppersberg JP, Veh R, Pongs O (1992) Characterization of a Shaw-related potassium channel family in rat brain. EMBO J 11:2473-2486.

Roth A, Häusser M (2001) Compartmental models of rat cerebellar Purkinje cells based on simultaneous somatic and dendritic patch-clamp recordings. J Physiol (Lond) 535:445-472.

Rudy B, McBain CJ (2001) Kv3 channels: voltage-gated $\mathrm{K}^{+}$channels designed for high-frequency repetitive firing. Trends Neurosci 24:517-526.

Ruppersberg JP, Stocker M, Pongs O, Heinemann SH, Frank R, Koenen M (1991) Regulation of fast inactivation of cloned mammalian $\mathrm{I}_{\mathrm{K}}(\mathrm{A})$ channels by cysteine oxidation. Nature 352:711-714.

Sharp AA, O'Neil MB, Abbott LF, Marder E (1993) The dynamic clamp: artificial conductances in biological neurons. Trends Neurosci 16:389-394.

Shieh C-C, Klemic KG, Kirsch GE (1997) Role of transmembrane segment S5 on gating of voltage-dependent $\mathrm{K}^{+}$channels. J Gen Physiol 109:767-778.

Soares D, Chitwood RA, Hyson RL, Carr CE (2002) Intrinsic neuronal properties of the chick nucleus angularis. J Neurophysiol 88:152-162.

Stuart GJ, Dodt H-U, Sakmann B (1993) Patch-clamp recordings from the soma and dendrites of neurons in brain slices using infrared video microscopy. Pflügers Arch 423:511-518.

Tansey EP, Chow A, Rudy B, McBain CJ (2002) Developmental expression of potassium-channel subunit Kv3.2 within subpopulations of mouse hippocampal inhibitory interneurons. Hippocampus 12:137-148.

Trussell LO (1997) Cellular mechanisms for preservation of timing in central auditory pathways. Curr Opin Neurobiol 7:487-492.

Vincent A, Lautermilch NJ, Spitzer NC (2000) Antisense suppression of potassium channel expression demonstrates its role in maturation of the action potential. J Neurosci 20:6087-6094.

Wang L-Y, Gan L, Forsythe ID, Kaczmarek LK (1998) Contribution of the Kv3.1 potassium channel to high-frequency firing in mouse auditory neurones. J Physiol (Lond) 509:183-194.

Wang X-J, Buzsáki G (1996) Gamma oscillation by synaptic inhibition in a hippocampal interneuronal network model. J Neurosci 16:6402-6413.

Weiser M, Bueno E, Sekirnjak C, Martone ME, Baker H, Hillman D, Chen S, Thornhill W, Ellisman M, Rudy B (1995) The potassium channel subunit KV3.1b is localized to somatic and axonal membranes of specific populations of CNS neurons. J Neurosci 15:4298-4314.

Whim MD, Kaczmarek LK (1998) Heterologous expression of the $\mathrm{K}_{\mathrm{v}} 3.1$ potassium channel eliminates spike broadening and the induction of a depolarizing afterpotential in the peptidergic bag cell neurons. J Neurosci 18:9171-9180.

Ylinen A, Bragin A, Nádasdy Z, Jandó G, Szabó I, Sik A, Buzsáki G (1995) Sharp wave-associated high-frequency oscillation $(200 \mathrm{~Hz})$ in the intact hippocampus: network and intracellular mechanisms. J Neurosci 15:30-46.

Zhang L, McBain CJ (1995a) Voltage-gated potassium currents in stratum oriens-alveus inhibitory neurones of the rat CA1 hippocampus. J Physiol (Lond) 488:647-660.

Zhang L, McBain CJ (1995b) Potassium conductances underlying repolarization and afterhyperpolarization in rat CA1 hippocampal interneurones. J Physiol (Lond) 488:661-672. 